\title{
The Possible Role Of Toll-Like Receptor 4 In The Pathology Of
}

\section{Stroke}

Elham Hakimizadeh ${ }^{1}$, M ohammad Allahtavakoli ${ }^{1,2 *}$, Ali Sham-sizadeh ${ }^{1,2}$, Ali Roohbakhsh ${ }^{3}$, Amir Moghadam-Ahmadi ${ }^{4}$, Mohammad Reza Rahmani ${ }^{1,2}$, Gholamreza Bazmandegan ${ }^{1,2}$

${ }^{1}$ Physiology-Pharmacology Research Center, Rafsanjan University of M edical Sciences, Rafsanjan, Iran.

${ }^{2}$ Dept. of physiology and pharmacology, Faculty of M edicine, Rafsanjan University of Medical Sciences, Rafsanjan, Iran.

${ }^{3}$ Pharmaceutical Research Center, School of Pharmacy, M ashhad University of Medical Sciences, Mashhad, Iran.

${ }^{4}$ Department of Neurology, School of Medicine, Rafsanjan University of Medical Sciences, Rafsanjan, Iran.

*Corresponding Author: Email: $\underline{m}$ _alahtavakoli@ rums.ac.ir

Stroke is a prevalent and dangerous health problem, which triggers an intense inflammatory response to Toll-like receptors (TLRs) activation. TLRs are the essential components of innate immunity system response, and therefore, they are one of the key factors involved in recognizing pathogens and internal ligands. Among TLRs, TLR4 significantly participates in the induction of inflammation and brain functions, hence, it has been hypothesized that this molecule is associated with several brain immunerelated diseases such as stroke. It has also been proved that animals with TLR4 deficiency have higher protection against ischemia and the absence of TLR4 reduces the neuroinflammation and injuries associated with brain trauma. TLR4 deficiency may play a neuroprotective role in the occurrence of stroke. This article will review recent information regarding the impact of TLR4 in the pathogenicity of stroke.

Key words: TLR4, Stroke, Inflammation

DOI: $10.7575 /$ aiac.abcmed.ca1.8

Published Date: February 2017

Peer-review is under responsibility of the 9th Iranian Stroke Congress.

Published by Australian International Academic Centre, Australia

This published work is open access under the CC BY license.

Available online at www.abcmed.aiac.org.au 